\title{
On the penetration of Alfven waves from the chromosphere into the corona
}

\author{
Yuri T. Tsap $\dagger$ \\ Crimean Astrophysical Observatory, Nauchny, Ukraine \\ email: yur@crao.crimea.ua
}

\begin{abstract}
Within the framework of the two-layer model the expression for the reflection coefficients of Alfven (torsion) waves propagated from the chromosphere into the corona have been obtained. As a result of reflection the energetic losses of waves with a period less than several tens of seconds are about $70 \%$. This shows evidence in favor of the essential contribution of Alfven waves with a period of $10-40$ s to coronal heating of the Sun.
\end{abstract}

Keywords. Sun: corona, chromosphere, waves, oscillations

There are many indications that Alfven waves excited in the lower atmosphere can be responsible for the high temperature of the solar corona. However the reflection of Alfven waves between the chromosphere and corona must be quite strong. Therefore the aim of this work is to consider the penetration of Alfven waves from the chromosphere into the corona on the basis of the two-layer model.

The linearized MHD equations described the propagation of Alfven waves (torsion modes) along the axis $\mathrm{Z}$ of the magnetic flux tube can be written as (Hollweg 1984)

$$
\rho \frac{\partial \delta v_{\varphi}}{\partial t}=\frac{B}{4 \pi} \frac{\partial \delta B_{\varphi}}{\partial z}, \quad \frac{\partial \delta B_{\varphi}}{\partial t}=B \frac{\partial \delta v_{\varphi}}{\partial z}
$$

where $\delta B_{\varphi}$ and $\delta v_{\varphi}$ are disturbances of the velocity and the magnetic field, respectively. Combining equations (1), we obtain the wave equation

$$
\frac{\partial^{2} \delta v_{\varphi}}{\partial t^{2}}=v_{A}^{2}(z) \frac{\partial^{2} \delta v_{\varphi}}{\partial z^{2}}
$$

where $v_{A}(z)=B / \sqrt{4 \pi \rho(z)}$ is the Alfven velocity.

In case where $v_{A} \propto \exp (z / 2 H)$ and $\delta v, \delta B \propto \exp (i \omega t)$ equation (2) has the following solution

$$
\delta v_{\varphi}=\left[C_{1} H_{0}^{(1)}(\eta)+C_{2} H_{0}^{(2)}(\eta)\right] e^{i \omega t} .
$$

where $C_{1}$ and $C_{2}$ are constants, $\eta=2 H \omega / v_{A}, H_{0}^{(1)}$ and $H_{0}^{(2)}$ are the Hankel functions.

Disturbances of velocities of the incident $\left(\delta v_{h}\right)$, reflected $\left(\delta v_{h}^{\prime}\right)$, and transmitted $\left(\delta v_{c}\right)$ waves are proportional to $H_{0}^{(1)}(\eta), H_{0}^{(2)}(\eta)$, and $e^{-i k z}$, respectively. Consequently, using the matching conditions at $z=0, \delta v_{h}+\delta v_{h}^{\prime}=\delta v_{c}, \delta B_{h}+\delta B_{h}^{\prime}=\delta B_{c}$, and (1), we find

$$
\frac{\delta v_{h}^{\prime}}{\delta v_{h}}=-\frac{1-i \omega /\left(k v_{A_{h}}\right) H_{1}^{(1)}(\eta) / H_{0}^{(1)}(\eta)}{1-i \omega /\left(k v_{A_{h}}\right) H_{1}^{(2)}(\eta) / H_{0}^{(2)}(\eta)} .
$$

$\dagger$ Present address: Crimean Astrophysical Observatory, Nauchny, 98409 Crimea, Ukraine. 


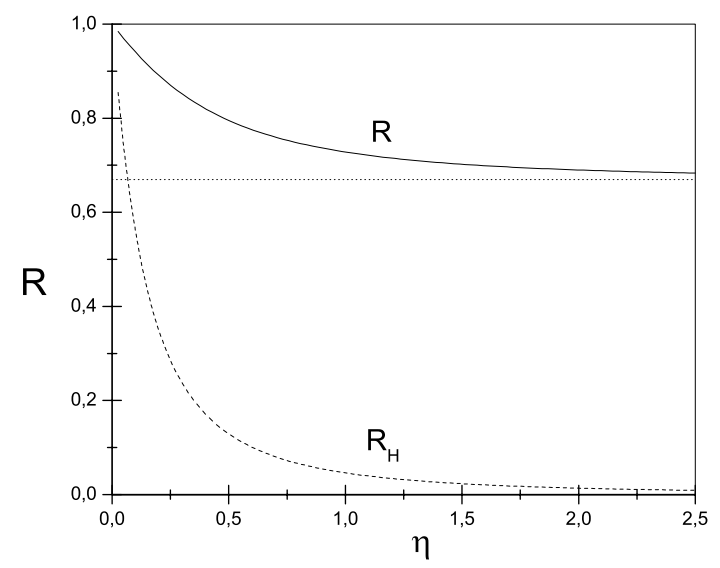

Figure 1. Plots of the reflection coefficients $R$ and $R_{H}$ versus $\eta$ at $\rho_{h} / \rho_{c}=10^{2}$.

It should be pointed out that equation (4) does not coincide with the appropriate formula (15) obtained by Hollweg (1984) (see also Leer et al. 1982). The main distinguish is connected with the ratio $\omega /\left(k v_{A_{h}}\right)$. Hollweg (1984) adopted $\omega /\left(k v_{A_{h}}\right)=1$ while as follows from our consideration $\omega /\left(k v_{A_{h}}\right)=\sqrt{\rho_{h} / \rho_{c}}$. It seems to us, equation (4) is more adequate since at $H \rightarrow \infty$ it is reduced to the well known expression, $\delta v_{h}^{\prime} / \delta v_{h}=$ $\left(v_{A_{c}}-v_{A_{h}}\right) /\left(v_{A_{c}}+v_{A_{h}}\right)$ (formula (15) gives $\delta v_{h}^{\prime} / \delta v_{h}=0$ ).

According to equation (4) the reflection coefficient is

$$
R=\left|\frac{\delta v_{h}^{\prime}}{\delta v_{h}}\right|^{2}=\frac{J_{0}(\eta)^{2}+N_{0}(\eta)^{2}+\left(\omega / k v_{A_{h}}\right)^{2}\left(N_{1}(\eta)^{2}+J_{1}(\eta)^{2}\right)-\left(\omega / k v_{A_{h}}\right) 4 /(\pi \eta)}{J_{0}(\eta)^{2}+N_{0}(\eta)^{2}+\left(\omega / k v_{A_{h}}\right)^{2}\left(N_{1}(\eta)^{2}+J_{1}(\eta)^{2}\right)+\left(\omega / k v_{A_{h}}\right) 4 /(\pi \eta)}
$$

where $J_{n}$ and $N_{n}$ are the Bessel and Macdonald functions, respectively. On the other side, Hollweg (1984) obtained

$$
R_{H}=\frac{J_{0}(\eta)^{2}+N_{0}(\eta)^{2}+N_{1}(\eta)^{2}+J_{1}(\eta)^{2}-4 /(\pi \eta)}{J_{0}(\eta)^{2}+N_{0}(\eta)^{2}+N_{1}(\eta)^{2}+J_{1}(\eta)^{2}+4 /(\pi \eta)} .
$$

Plots of $R(\eta)$ and $R_{H}(\eta)$ are shown in Fig. 1. It is seen that at $\eta>\eta_{0} \approx 1$ the reflection coefficient $R \approx 0.7$ is not significantly changed. Therefore the transmission coefficient can be evaluated as $T=1-R \approx 0.3$. Taking $v_{A_{h}}=3 \times 10^{7} \mathrm{~cm} / \mathrm{s}$, we obtain the characteristic period $T_{p}=4 \pi H /\left(\eta_{0} V_{A_{h}}\right) \approx 21 \mathrm{~s}$. Such oscillations have been observed both in microwave and in optical ranges (e.g. Gelfreikh et al. 2004). Note especially that Alfven waves with $T_{p}<10 \mathrm{~s}$ are strongly damped in the partially ionized plasma of the solar chromosphere (De Pontieu et al. 2001).

Thus, for reasons given above we can suggest that the short-period Alfven waves with $T_{p}=10-40 \mathrm{~s}$ may play essential role in coronal heating.

\section{References}

De Pontieu, B., Martens, P.C. \& Hudson, H.S. 2001. Astrophys. J 558, 859

Gelfreikh, G.B., Tsap, Y.T., Kopylova, Y.G. et al. 2004, Astron. Lett. 30,489

Hollweg, J.V. 1984, Astrophys. J. 277, 392

Leer, E., Holzer, T.E. \& Flå, T. 1982, Space Sci. Rev. 33, 161 